\title{
Experiencia educativa con el uso de la plataforma Moodle
}

Francisco Jaramillo González.

C onocer desde hace varios años acerca de los usos educativos de la computadora, el avance vertiginoso de las tecnologías de información y comunicación (TIC) y su incorporación en la educación, determinando un cambio hacia nuevos modelos de enseñanza, aunado a que nuestros estudiantes están inmersos en la era digital, me planteó la necesidad de capacitarme en el conocimiento de las TIC y su incorporación en el quehacer docente. Gracias al programa de educación continua del "Diplomado en Educación a Distancia para Profesores" he cubierto esa necesidad.

El resultado ha sido la elaboración de un Proyecto de trabajo educativo para la enseñanza de la Anatomía Humana y Morfología del Sistema Nervioso diseñado para estudiantes del primer y segundo semestre respectivamente, de la carrera de Medicina de nuestra Universidad, con el apoyo de un ambiente de aprendizaje interactivo basado en el uso de la plataforma Moodle ${ }^{1}$ instalada en el servidor de la Institución como complemento de la enseñanza presencial de estas asignaturas.

Disponer de una plataforma educativa como Moodle ha posibilitado la incorporación de modelos más adecuados en la enseñanza y aprendizaje de la Anatomía y de prácticamente todas las áreas del conocimiento, apoyados en la utilización de nuevas tecnologías maximizando la utilización de recursos en el proceso de construcción del conocimiento. Dicha experiencia se está concretizando en nuestra Universidad y se ha fortalecido a partir de la implementación del Modelo Educativo Institucional que tiene como uno de sus objetivos una educación de calidad, incorporando nuevas propuestas psicopedagógicas y tecnológicas, y que en los últimos años se han comenzado a realizar experiencias relacionadas con la introducción de las TIC en educación a distancia y como apoyo a la enseñanza presencial.

Con base en estos planteamientos, desde hace cuatro años he desarrollado e implementado un proyecto de innovación basado en aplicar una alternativa de estrategia metodológica concretamente en las asignaturas señaladas, desde un enfoque constructivista, con el propósito de proporcionar a los estudiantes las herramientas básicas de las TIC con el fin de optimizar la adquisición y el manejo de información efectiva y eficiente a través del uso de la plataforma educativa Moodle como un complemento a la educación presencial.

El proyecto está basado desde sus inicios en la aplicación de una estrategia metodológica capaz de propiciar procesos de enseñanza y aprendizaje centrados en la construcción del conocimiento de la anatomía humana por parte del estudiante de medicina, mediante la incorporación de las tecnologías de información y comunicación como una fórmula

Moodle (Entorno de Aprendizaje Dinámico Orientado a Objetos y Modular) es una plataforma de aprendizaje basada en principios pedagógicos que promueven en los estudiantes la responsabilidad de su aprendizaje, y facilita la comunicación sincrónica y asincrónica de todos los participantes, además de propiciar un cambio en el trabajo docente al aplicar una metodología de enseñanza que favorece el aprendizaje de los estudiantes, al promover el trabajo colaborativo, facilitarles la disposición de material didáctico, tutoría, entre otros. 
alternativa y complementaria a la docencia presencial, en el desarrollo de habilidades necesarias para utilizar las TIC en la aplicación de estrategias, métodos activos de aprendizaje y actividades de formación a través de Internet, que propicien el autoaprendizaje de los estudiantes y promuevan el trabajo cooperativo, y por lo tanto la aplicación de nuevas metodologías de enseñanza activa, interactiva y participativa mediante la utilización de la plataforma educativa Moodle como herramienta para colocar recursos de información, y desarrollar actividades para los estudiantes, tendientes a la construcción de aprendizajes significativos.

La alternativa de utilizar la plataforma Moodle como complemento en la enseñanza presencial de la anatomía generó un cambio de actitud profesor-estudiante hacia los procesos de enseñanza y aprendizaje de esta disciplina. Propiciar procesos tendientes a una mayor interactividad de los estudiantes con los materiales didácticos favoreció la construcción de su aprendizaje al contar con el apoyo de múltiples recursos tecnológicos que les permitieron la utilización sistemática de imágenes, animaciones, atlas interactivos, software educativo, video, entre otras herramientas, unidas a los recursos disponibles en el laboratorio, como modelos anatómicos tridimensionales, piezas anatómicas, videos, placas de imagenología y el trabajo con cadáver.

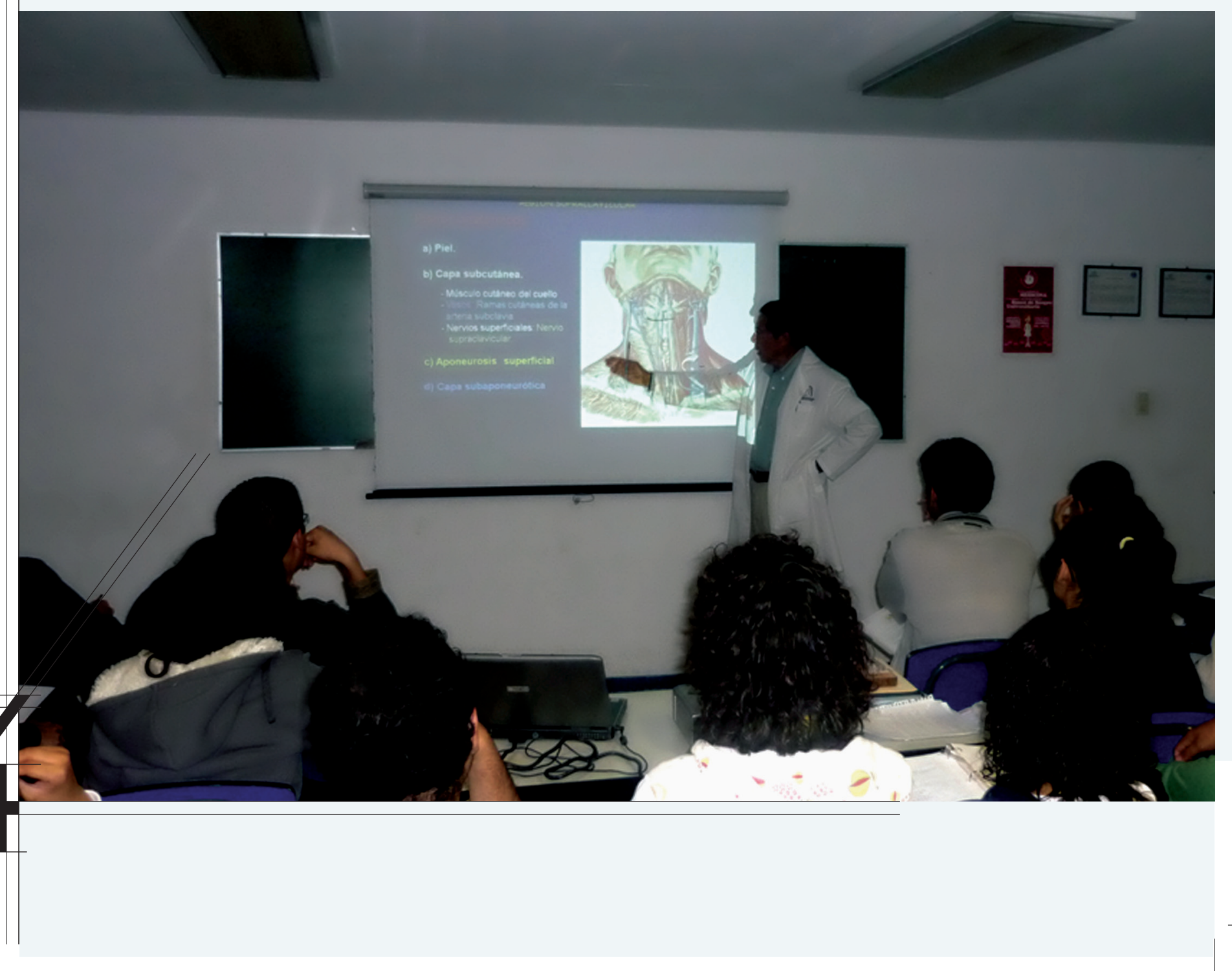


Esta propuesta educativa permitió combinar y complementar la enseñanza presencial con la modalidad no presencial tomando de cada modelo las ventajas para un mejor aprendizaje, favoreciendo el trabajo docente y de los estudiantes. Como docente me ha permitido planificar los cursos e incorporar múltiples materiales de apoyo en la plataforma, así como programar actividades individuales y grupales con el fin de reforzar en los estudiantes los conocimientos adquiridos. De igual forma, los estudiantes, al trabajar en una plataforma educativa, adquieren una visión diferente respecto a las sesiones presenciales, aprenden y se fomenta el trabajo colaborativo, interactúan con el profesor y sus compañeros de manera asincrónica a través de las múltiples herramientas que la plataforma provee. Por lo anterior, se puede resumir que la importancia pedagógica de utilización de la plataforma Moodle radica en que posibilita una mejor atención y relación profesor-estudiante, aprovechamiento de los recursos que nos ofrece Internet, implementación de una innovación educativa, y por lo tanto, una mejor calidad de la enseñanza.

La experiencia me ha permitido obtener información sobre la opinión de los estudiantes sobre la metodología, actividades y materiales empleados al incorporar las TIC en el proceso educativo. Los resultados obtenidos indican que a pesar de que los estudiantes valoraron positivamente la utilización de la plataforma Moodle, la evaluación sumativa, aunque ha mejorado, no ha sido muy significativa respecto a los cursos en que no se contó con esta plataforma; sin embargo, aunque aparentemente no hubo diferencias sustanciales cuantitativamente hablando, la diferencia radicó en el cambio de actitud hacia los procesos de enseñanza y aprendizaje, ya que de acuerdo con la evaluación global de la experiencia docente y del aprendizaje, se favoreció la adquisición de capacidades que enmarcan la educación fundamental: "aprender a saber", "aprender a hacer", "aprender a socializar" y "aprender a ser". ${ }^{2}$ Por lo anterior, esta propuesta educativa implicó un cambio, no sólo en las formas tradicionales de la enseñanza y el aprendizaje de la anatomía, sino también en fomentar una cultura de autoaprendizaje y aprendizaje colaborativo, al promover que el estudiante participe activamente en la construcción de sus conocimientos.

El aprendizaje logrado con esta experiencia educativa, al poner en práctica principios pedagógicos que posibilitaron la construcción e implementación del uso de la plataforma educativa en la enseñanza presencial de la anatomía, me ayuda a reflexionar que los profesores debemos tener una apertura ante las nuevas posibilidades pedagógicas que nos ofrecen las TIC y, por lo tanto, debemos desarrollar competencias en la formación y actualización para aprovechar las grandes posibilidades de uso pedagógico que tienen dichas tecnologías.

Por otra parte, debemos tener en cuenta que la nueva tecnología no garantiza el éxito pedagógico si su aplicación no se sustenta en una apropiada metodología, ya que existe una serie de aspectos que deben cumplirse para lograr implementar debidamente esta modalidad educativa, por lo que su utilización debe estar cimentada en una metodología didáctica que permita aplicar principios pedagógicos que posibiliten en el estudiante la participación en la construcción de su conocimiento, ya que su adecuado diseño e implementación motiva y facilita el desarro- 
son imperativos cambios en el diseño curricular, capacitación de profesores y estudiantes, que redunden en una educación de alta calidad. ${ }^{3}$

El desafío para el docente en esta época de grandes avances tecnológicos, como lo señala Scagnoli, ${ }^{4}$ es capacitarse en el conocimiento de la tecnología y en el adecuado diseño de los objetivos de aprendizaje que se pretenden lograr al utilizar las TIC como complemento de su clase presencial, que le permitan diseñar este tipo de actividades con el fin de planificar de manera pertinente la experiencia de aprendizaje, y por lo tanto permitirle al docente cumplir con un rol que lo habilite en el aprovechamiento de dichas tecnologías con fines educativos, con el objetivo de lograr que los estudiantes alcancen las habilidades necesarias para el uso eficiente de los recursos que nos proporcionan estas tecnologías.

Esta experiencia de innovación educativa llevada a cabo mediante la utilización de la plataforma Moodle ha sido para mí muy gratificante, sobre todo, porque con base en la valoración realizada a los estudiantes intuyo que también lo ha sido para ellos, lo cual me motiva a seguir invirtiendo tiempo y esfuerzo para lograr aprovechar esta herramienta tecnológica con el fin de mejorar sustancialmente el aprovechamiento del aprendizaje por parte de los estudiantes.

\section{Fuentes de consulta}

Aznar Díaz, I. y Francisco Javier Hinojo, "Una experiencia basada en el uso de recursos pedagógicos desarrollados a partir de la nuevas tecnologías: aplicación de la metodología Blended e-Learning en el contexto universitario", 2006. Recuperado en marzo de 2008 en http://www.educaweb.com/EducaNews/interface/asp/web/NoticiesMostrar.asp?NoticiaID=1216\&SeccioID=1670

Fernandez, G.J., "La plataforma educativa Moodle. La hora del eaprendizaje", 2005. Recuperado en febrero de 2006 en http://www. linux-magazine.es/issue/13/Educacion.pdf

Ibáñez, J.E., “El uso educativo de las TIC”. Recuperado en marzo de 2006 en http://www.pangea.org/jei/edu/f/tic-uso-edu.htm\#_Toc50017373

Jaramillo, G.F., Aplicación de las Tecnologías de Informática y Comunicación como complemento en la enseñanza presencial de la anatomía. Proyectos de Innovación 2006, UAA, 2008.

Marqués, P.G., "Impacto de las TIC en educación: Funciones y limitaciones". Recuperado en febrero de 2007 en http://dewey.uab.es/ pmarques/siyedu.htm\#porque

Scagnoli, Norma I., "El Aprendizaje Colaborativo en Cursos a Distancia" Rev. Investigación y Ciencia, UAA, Núm. 36, septiembrediciembre 2006.

World Conference on Higher Education. "Higher Education in the Twenty-first Century. Vision and Action", Unesco, París, 5-9 octubre 1998, Recuperado en febrero de 2007 en http://unesdoc.unesco. org/images/0011/001164/116428e.pdf 